\title{
Teratoma of the adrenal gland: clinical experience and literature review
}

\author{
Cheng He ${ }^{1,2 \#}$, Yanli Yang ${ }^{1 \#}$, Yang Yang ${ }^{1}$, Fuqin Wang ${ }^{1}$, Jie $\mathrm{Hu}^{1}$, Jingjing Zhang ${ }^{1}$, Yu Yin ${ }^{1}$, Lili Zeng ${ }^{3}$, \\ Tijiang Zhang ${ }^{1}$, Heng Liu ${ }^{1}$ \\ ${ }^{1}$ Department of Radiology, Affiliated Hospital of Zunyi Medical University, Zunyi, China; ${ }^{2}$ Medical Imaging Department, Chongqing University \\ Central Hospital, Chongqing, China; ${ }^{3}$ Department of Pathology, Affiliated Hospital of Zunyi Medical University, Zunyi, China \\ \#These authors contributed equally to this work. \\ Correspondence to: Dr. Heng Liu; Dr. Tijiang Zhang. Department of Radiology, Affiliated Hospital of Zunyi Medical University, Medical Imaging \\ Center of Guizhou Province, Zunyi, China. Email: zmcliuh@163.com; tijzhang@163.com; Dr. Lili Zeng. Department of Pathology, Affiliated \\ Hospital of Zunyi Medical University, Zunyi, China. Email: 358753897@qq.com.
}

\begin{abstract}
Teratoma originates from pluripotent cells of two or more than two germ cell layers, and most of them are benign. Teratomas are found in the ovaries and testes. Retroperitoneal teratoma is rare, especially adrenal teratoma. Here, we describe a rare case of a 17-year-old woman who was diagnosed with pulmonary tuberculosis and a right adrenal mass at the age of eight. So, she received anti-tuberculosis treatment. Nine years later, chest X-rays showed prior lesions in both lungs, and abdominal CT showed the mass in the right adrenal gland was larger than before, during this period she had no clinical symptoms. She underwent retroperitoneal laparoscopic adrenalectomy, and the pathological diagnosis was a mature teratoma of the right adrenal gland. During a one-year follow-up, the patients recovered well without any discomfort. Thirty-two cases were found in the literature review, among which no patients had a history of pulmonary tuberculosis. Adrenal teratoma is often seen in females and the left adrenal gland. The imaging features of adrenal teratoma can be cystic, solid, and cystic solids. Mature fat and calcification can be seen in most teratomas. Comprehensive analysis of clinical features and imaging characteristics can enhance the diagnostic confidence of radiologists in adrenal teratoma.
\end{abstract}

Keywords: Case report; adrenal teratoma; tuberculosis; imaging

Submitted Jul 07, 2020. Accepted for publication Aug 10, 2020.

doi: 10.21037 /gs-20-648

View this article at: http://dx.doi.org/10.21037/gs-20-648

\section{Introduction}

Teratomas are most common from ovaries and testes, and patients usually have no overt clinical symptoms. Most teratomas are found in health examinations or gynecological examinations. Teratoma can also occur in the mediastinum, intracranial, sacrococcyx, and retroperitoneal space. Nevertheless, retroperitoneal teratoma is rare, especially adrenal teratoma, the incidence rate of which is $0.13 \%(1,2)$. The pathological classification of teratoma includes mature teratoma and immature teratoma. Mature teratoma means that there are no malignant and immature components in the tumor, and there are no similar lesions in other parts of the body (3). About $1.5-2 \%$ of teratoma is malignant. Immature teratoma contains immature embryonic tissue, about $26 \%$ of which is malignant (4).

The imaging characteristics of adrenal teratoma are partly like those of adrenal angiomyolipoma, myelolipoma, and adenoma $(5,6)$. It is difficult to diagnose before the operation $(7,8)$. Here, we describe a rare case of a 17 -year-old woman who was diagnosed with pulmonary tuberculosis and right adrenal mass at the age of eight. Nine years later, she underwent retroperitoneal laparoscopic adrenalectomy with a pathological diagnosis of a right adrenal teratoma. We summarized the clinical 
manifestations and CT characteristics of adrenal teratoma and conducted a review of the relevant literature, and the information is summarized in Table 1.

We present the following article in accordance with the CARE reporting checklist (available at http://dx.doi. org/10.21037/gs-20-648).

\section{Case presentation}

The 17-year-old Chinese woman first appeared in our hospital in July 2010, when she was only eight years old. CT showed a spherical mass of about $3.2 \mathrm{~cm} \times 3.2 \mathrm{~cm}$ $\times 4.0 \mathrm{~cm}$ in the right adrenal region, with a clear and smooth boundary and multiple calcifications in the mass. The radiologist diagnoses adrenal myelolipoma or neuroblastoma, and she also suffers from tuberculosis in the middle lobe of the right lung and Upper lobes of both lungs (Figure 1). One month after anti-tuberculosis treatment, chest $\mathrm{CT}$ showed the absorption of pulmonary lesions decreased, and there was no change in the right adrenal gland mass.

She reappeared in our hospital in February 2019, during this period she had no clinical symptoms. CT showed a mixed density mass of about $5.2 \mathrm{~cm} \times 4.2 \mathrm{~cm} \times 5.6 \mathrm{~cm}$ in the right adrenal region with a CT value of -133 to 2,169 HU. The mass has fat, soft tissue, and calcification. The three-dimensional reconstruction showed that there was a tooth-like high-density shadow in the mass, and the soft tissue components were enhanced on a contrast-enhanced scan. Chest X-rays showed multiple patchy and nodular high-density shadows in both lungs (Figure 1). Laboratory tests showed that norepinephrine: $1,174.0 \mathrm{pg} / \mathrm{mL}$ (normal range $<600 \mathrm{pg} / \mathrm{mL}$ ), and adrenaline, dopamine, supine position, aldosterone, and standing position aldosterone were all normal. Ferritin: $10.2 \mu \mathrm{g} / \mathrm{L}$ (normal range 11.0-306.8 $\mu \mathrm{g} / \mathrm{L}$ ), and carbohydrate antigen 19-9, carbohydrate antigen 153 , carbohydrate antigen 125, alpha-fetoprotein, carcinoembryonic antigen were all normal. She then underwent retroperitoneal laparoscopic adrenalectomy under general anesthesia and the tumor was completely removed. The vital signs of the patients were stable during the operation, the patients received hemostasis and symptomatic support treatment after operation, and the incision healed in one stage. The postoperative gross pathology showed that the tumor was gray white cystic with a size of $7.0 \mathrm{~cm} \times 2.5 \mathrm{~cm} \times 2.0 \mathrm{~cm}$, containing hair, teeth and bean curd residue like substances. Microscopically, skin, sweat glands, hair follicles, sebaceous glands and adrenal tissues were seen. Pathological diagnosis was mature teratoma (Figure 2). Postoperative CT showed that the right adrenal teratoma was resected entirely, and no tumor recurrence or metastasis was found. During a oneyear follow-up, the patients recovered well without any discomfort.

The ethics committee approved this study of The Affiliated Hospital of Zunyi Medical University, and informed consent was obtained from the patient for the anonymous use of the clinical, imaging, and histologic data for publication. All procedures performed in this study involving human participants were in accordance with the Declaration of Helsinki (as revised in 2013).

\section{Literature review}

PubMed, Medline, Web of Science, and Ovid databases were searched for English-language case reports and case series of adrenal teratoma published in the last 30 years from April 1990 to March 2020. The following keywords were used: (Adrenal Glands or Adrenal Gland or Gland Adrenal or Glands Adrenal) and (Teratoma or Teratomata). The flow chart of the literature screening process is set out in Figure 3. A total of 26 full-text articles involving 32 cases were included for analysis (Table 1).

According to the literature, before our case, only 32 cases, including 34 adrenal teratoma lesions, were reported in English-langue. Adrenal teratoma patients are mainly adolescents with an average age of about 27 years. The adrenal-related hormones are normal and most of them had no clinical symptoms. Adrenal teratomas are unilateral, one case with bilateral adrenal involvement. Adrenal teratoma is often seen in females and the left adrenal gland. It should be noted that imaging features of adrenal teratoma can be cystic, solid, or cystic solid. Mature fat and calcification or bone tissue can be observed in most teratomas (Figure 4). Most adrenal teratomas are benign, mature teratomas, two with focal carcinoid foci. Fifteen of the 36 patients were followed up for 3 months to 4 years, with an average followup time of about 1 year. All patients had a good prognosis and no tumor recurrence or other discomfort.

\section{Discussion}

Teratoma is a type of germ cell tumor originating from peripheral pluripotent stem cells, which composed of tissues from the ectoderm, mesoderm, and endoderm tissues. The tumor may be solid, unicystic, multilocular, or cystic solid, 


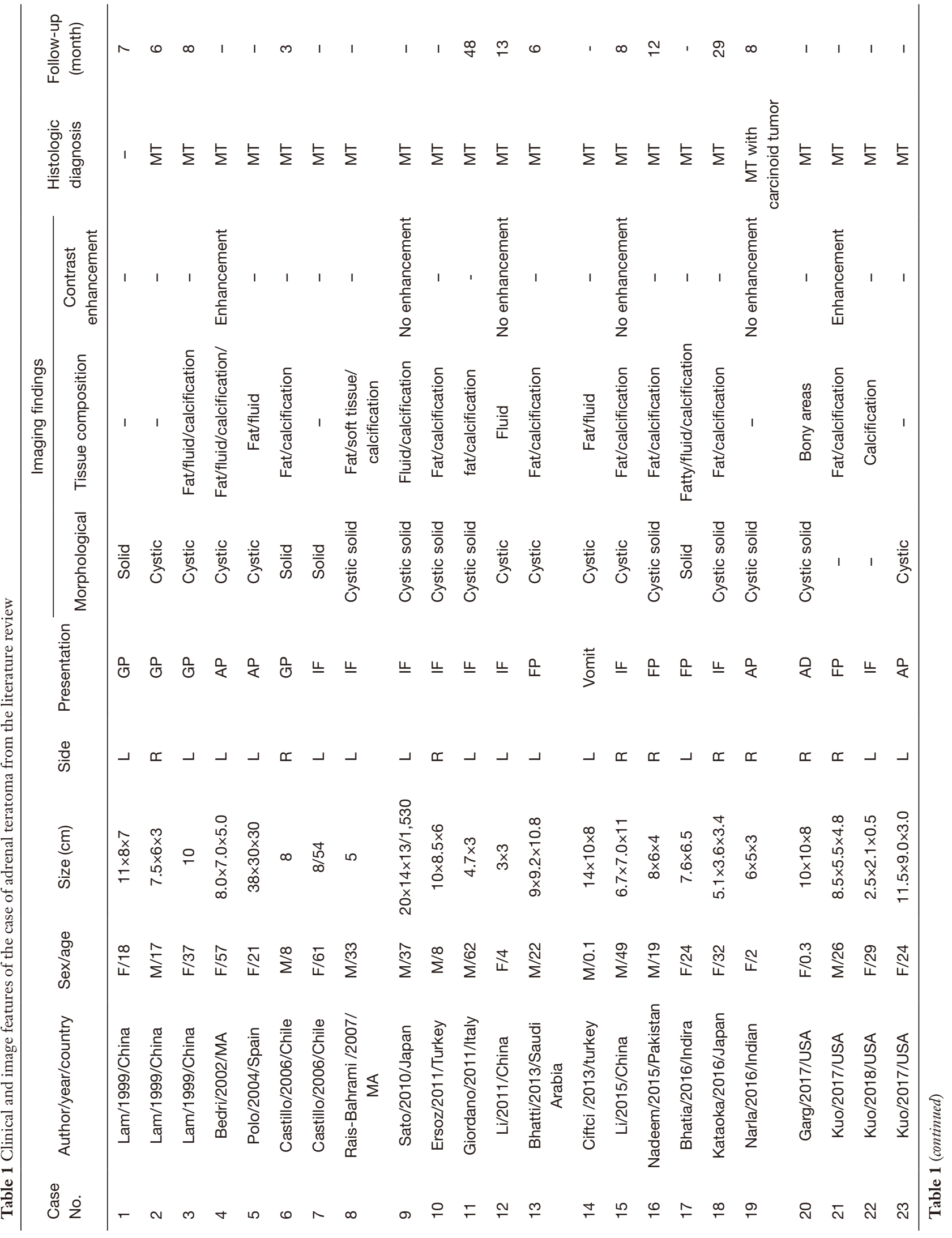




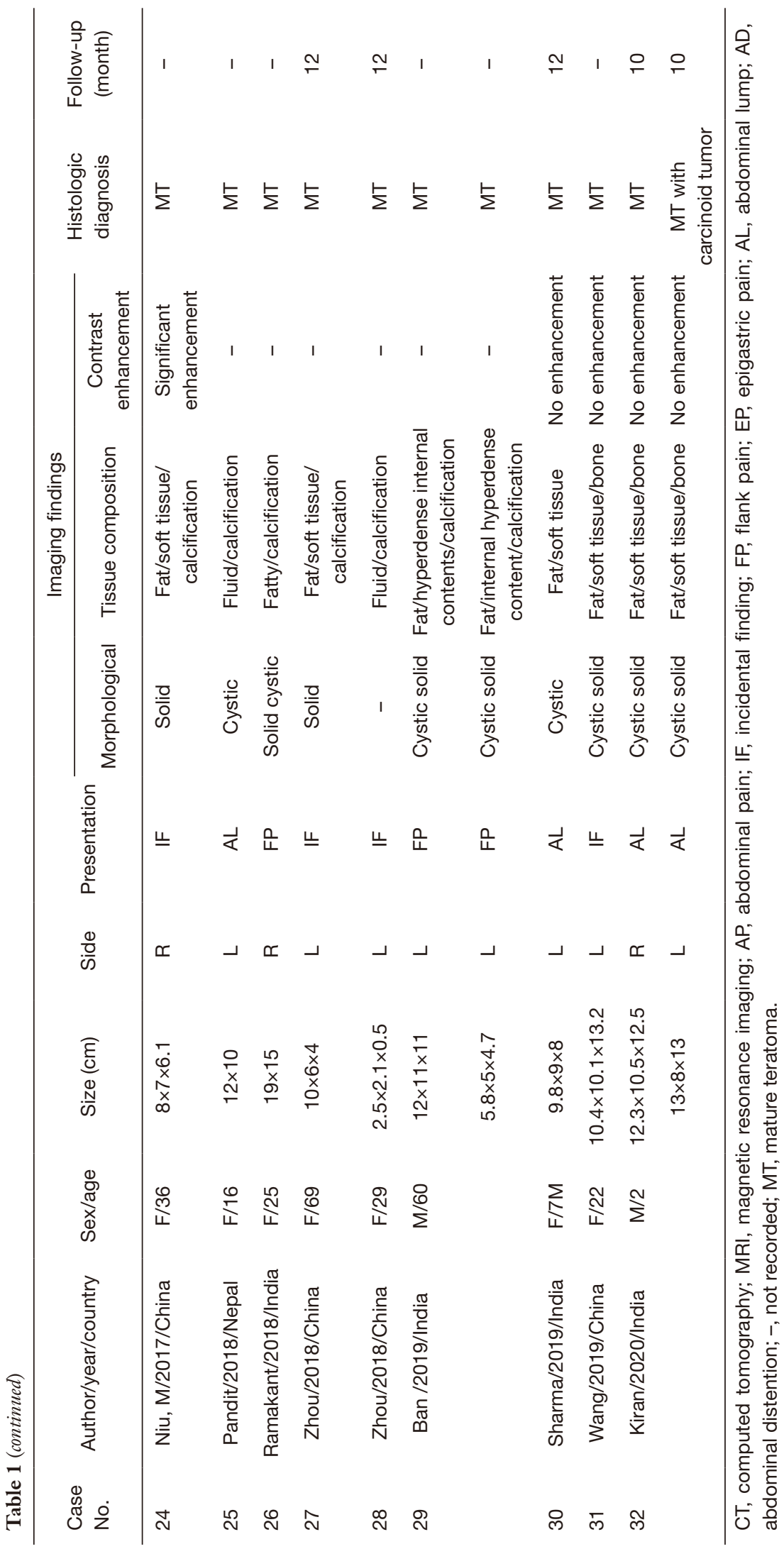



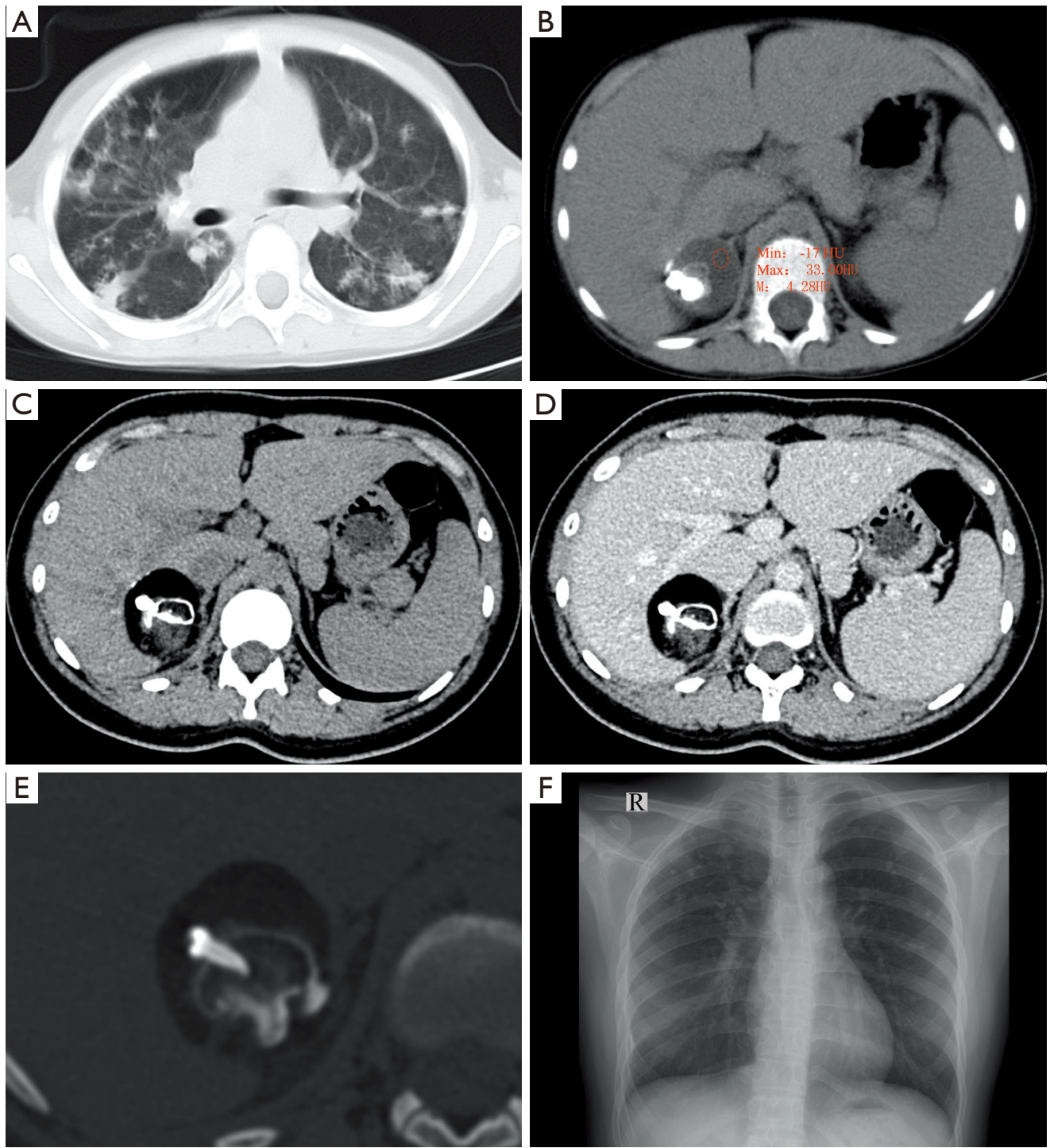

Figure 1 (A,B) A 17-year-old women at the age of 8. (A) Multiple patchy and nodular high-density shadows in the middle lobe of the right lung and upper lobes of both lungs, some of which were accompanied by punctate calcification [window width (WW) 1,382, window level (WL) -360]. (B) A spherical mass in the right adrenal region, with predominantly liquid density and multiple calcifications in the mass (WW 246, WL 65). (C,D,E,F) A 17-year-old women, CT image of upper abdomen. (C) Right adrenal mixed density mass with fat, soft tissue and calcification (WW 220, WL 40); (D) enhanced scan showed mild enhancement of soft tissue in the mass (WW 220, WL 40); (E) threedimensional reconstruction showed there was tooth-like high-density in the mass (WW 2,612, WL 960); (F) chest X-ray, multiple patchy and nodular high-density shadows in both lungs.

containing hair, sebum, cystic fluid, calcification, or teeth (9). Teratomas are predominantly located in the gonads (testis or ovaries), $15 \%$ occurring in the extragonadal, $4 \%$ in the retroperitoneum, the adrenal gland is even rarer (10). According to the literature review, 32 cases of adrenal teratoma were reported in English-language literature before this. Patients with adrenal teratoma usually have no clinical manifestations. Additionally, some patients presented with low back pain, abdominal mass, upper abdominal pain, among many others. Adrenal-related endocrine tests are usually regular.

The imaging characteristics of adrenal teratomas are like 

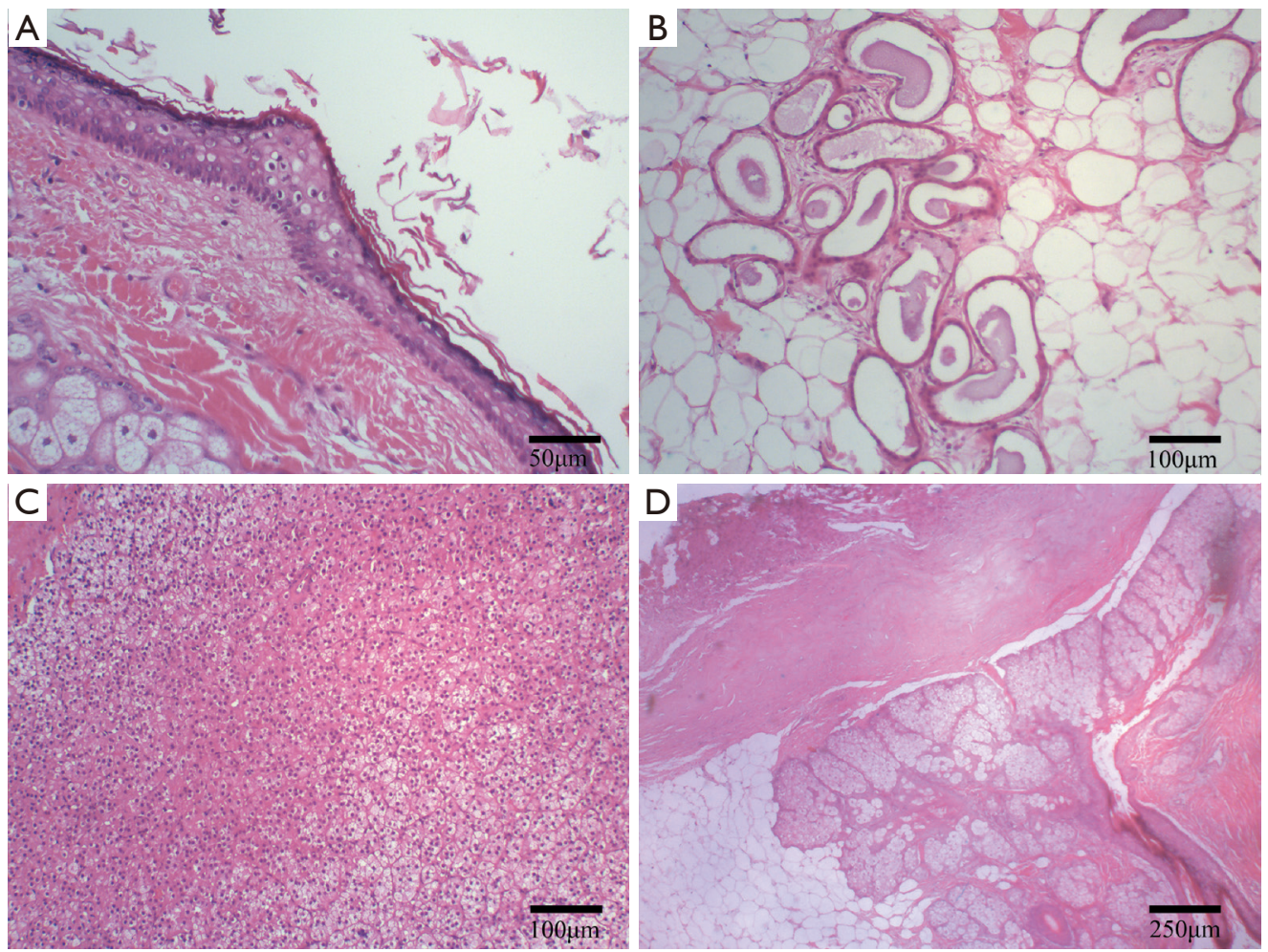

Figure 2 A 17-year-old women, microscopic examination of the mass. (A) Skin [hematoxylin and eosin (H\&E) staining, magnification, $\times 200$ ]; (B) sweat gland (H\&E staining, magnification, $\times 100)$; (C) adrenal gland and keratin (H\&E staining, magnification, $\times 100)$; (D) adrenal and sebaceous gland (H\&E staining, magnification, $\times 40)$.

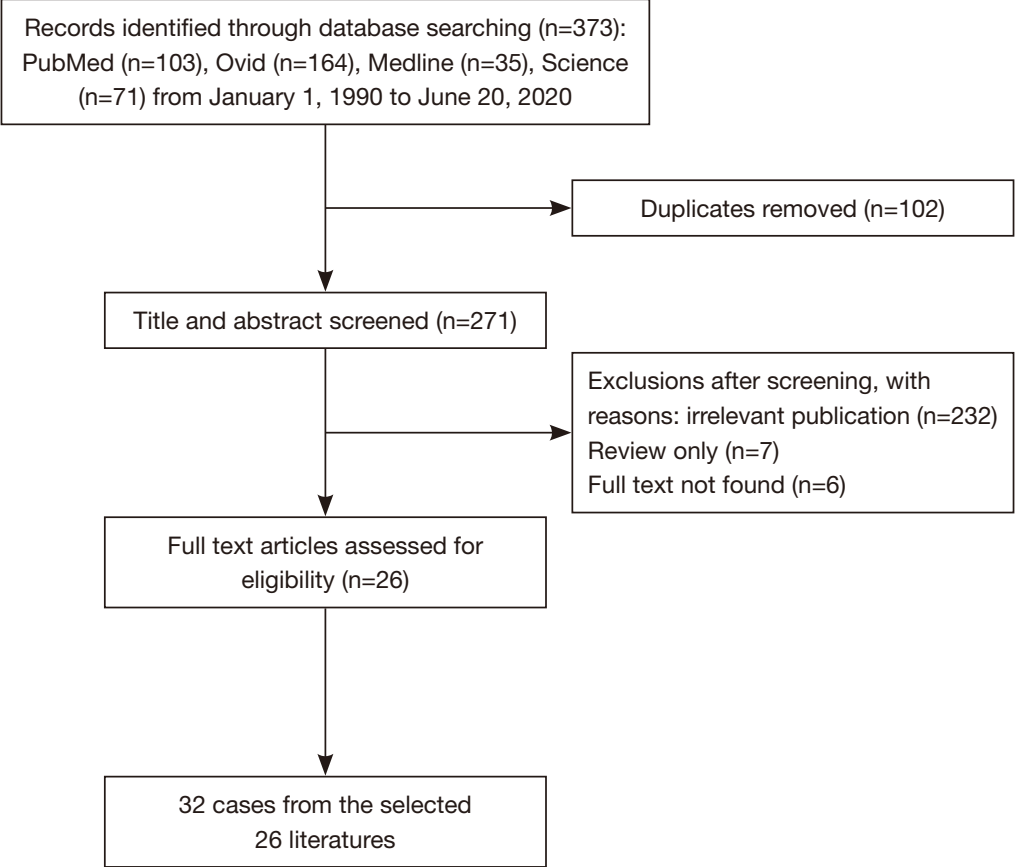

Figure 3 Flow chart of the literature screening process for adrenal teratoma. 


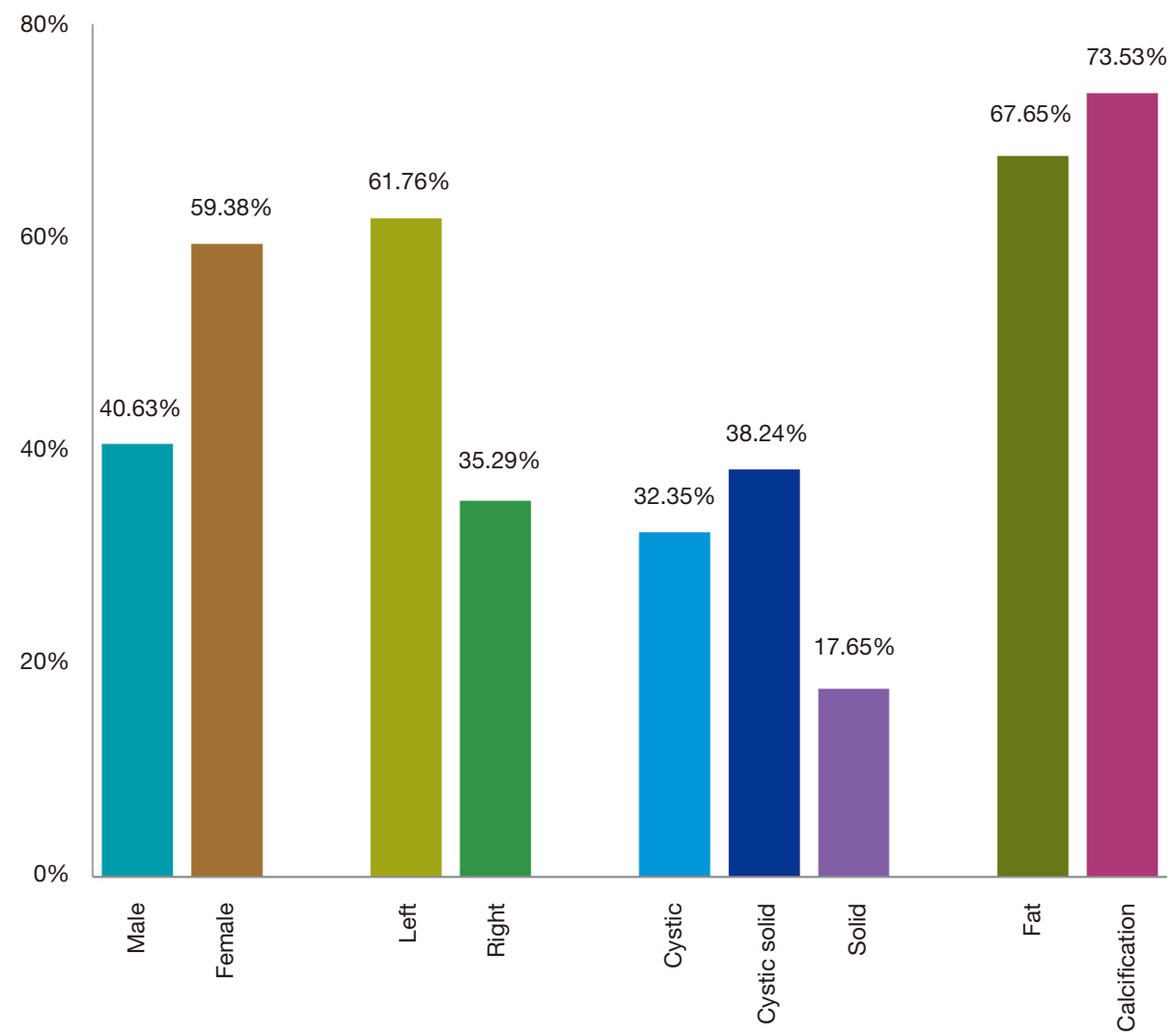

Figure 4 Clinical distribution and imaging characteristics of adrenal teratoma. Adrenal teratoma is often seen in females and the left adrenal gland. The imaging features of adrenal teratoma can be cystic, solid, or cystic solid. Mature fat and calcification can be seen in most teratomas.

those teratomas in other locations, most of which present as mixed density masses with predominant fat density/ signal, and contain calcification, bone, teeth, soft tissue, and fluid components. Calcification can be punctate, radial, and linear. Soft tissue components can be accompanied by varying degrees of enhancement. The most critical preoperative diagnosis of adrenal teratoma is to differentiate it from other adrenal diseases with fat and calcification.

\section{Differential diagnosis of adrenal teratoma and other fatty tissue containing lesions}

Adrenal adenomas are the most common adrenal tumors, and about $70 \%$ of adenoma cells contain intracellular lipids $(11,12)$. Adrenal adenomas are divided into functional and non-functional types. Functional adenomas are usually less than $3 \mathrm{~cm}$ with adrenal hormone abnormalities. The volume of non-functional larger adenoma, and the larger adenoma, the more likely there is a hemorrhage, necrosis, and cystic degeneration. Most adrenal adenomas show uniform watery density, clear boundary on CT, and mild enhancement on the contrast-enhanced scan. The signal intensity of the adrenal adenoma on MR is like the liver, the signal on the out-phase image is significantly decreased, and the enhanced scan showed slight enhancement (13).

The most common adrenal lesion containing mature adipose tissue is myelolipoma, a benign tumor containing bone marrow components $(14,15)$. Adrenal myelolipoma has no endocrine function and is often found incidentally during physical examination (16). Adrenal myelolipoma presents as a soft tissue mass, and with fat density on CT, $20 \%$ of which accompanied by calcification, and the soft tissue part is enhanced. The adipose component shows a high signal on both T1WI and T2WI on MR, while bone marrow shows a low signal on T1WI and isosignal on T2WI, the signal on fat suppression image decreases significantly. There is no signal decreasing on in-phase and out-phase images. 
Adrenal angiomyolipoma is also rare. The content of these components determines the imaging findings of adrenal angiosarcoma. The larger ones $(>4 \mathrm{~cm})$ may be accompanied by scattered calcification, and the enhanced scan shows significant enhancement (17). The calcification of adrenal teratoma is lamellar, annular, or linear, and the teratoma has no enhancement or mild enhancement, which can supply the basis for the differential diagnosis of radiologists.

\section{Differential diagnosis of adrenal teratoma and other calcified lesions}

Metastases are the most common malignant tumors of the adrenal gland, and adrenal metastases from different sources have different imaging characteristics. Metastases from colon, breast, and small cell lung cancers can be accompanied by calcification and need to be differentiated from adrenal teratomas $(18,19)$. Patients with adrenal metastases usually have a history of primary tumors, and most are multiple metastases with bilateral adrenal involvement. The most crucial distinguishing point between adrenal metastases and teratomas is whether the lesion has adipose tissue or not.

In the case we reported, the patient had a history of tuberculosis, so the possibility of adrenal tuberculosis must be considered. Adrenal tuberculosis is often caused by blood invasion of Mycobacterium tuberculosis. The clinical manifestations are low fever, fatigue, night sweats, adrenal dysfunction, among many others (20). The CT features of adrenal tuberculosis are bilateral adrenal enlargement, irregular shape, and high density (21). When there is caseous necrosis, the density of the mass is uneven and may be accompanied by an insufficient amount of calcification, complicated with large retroperitoneal lymph nodes, and circular enhancement. In the later stage, the whole adrenal gland was calcified, or part of the cheese was calcified. Contrast-enhanced scans often showed marginal enhancement, low-density casein necrosis in the center, or inhomogeneous enhancement (22).

\section{Conclusions}

In conclusion, primary adrenal teratomas are exceedingly rare. Imaging examination showed an adrenal mass with mature fat and irregular calcification or teeth, which had high value in the diagnosis of an adrenal teratoma. Comprehensive analysis of clinical features and imaging characteristics can enhance the diagnostic confidence of radiologists in adrenal teratoma.

\section{Acknowledgments}

Funding: None.

\section{Footnote}

Reporting Checklist: The authors have completed the CARE reporting checklist. Available at http://dx.doi.org/10.21037/ gs-20-648

Conflicts of Interest: All authors have completed the ICMJE uniform disclosure form (available at http://dx.doi. org/10.21037/gs-20-648). The authors have no conflicts of interest to declare.

Ethical Statement: The authors are accountable for all aspects of the work in ensuring that questions related to the accuracy or integrity of any part of the work are appropriately investigated and resolved. The ethics committee approved this study of The Affiliated Hospital of Zunyi Medical University, and informed consent was obtained from the patient for the anonymous use of the clinical, imaging, and histologic data for publication. All procedures performed in this study involving human participants were in accordance with the Declaration of Helsinki (as revised in 2013).

Open Access Statement: This is an Open Access article distributed in accordance with the Creative Commons Attribution-NonCommercial-NoDerivs 4.0 International License (CC BY-NC-ND 4.0), which permits the noncommercial replication and distribution of the article with the strict proviso that no changes or edits are made and the original work is properly cited (including links to both the formal publication through the relevant DOI and the license). See: https://creativecommons.org/licenses/by-nc-nd/4.0/.

\section{References}

1. Sun H, Ding H, Wang J, et al. The differences between gonadal and extra-gonadal malignant teratomas in both genders and the effects of chemotherapy. BMC Cancer 2019;19:408.

2. Li S, Li H, Ji Z, et al. Primary adrenal teratoma: Clinical characteristics and retroperitoneal laparoscopic resection 
in five adults. Oncol Lett 2015;10:2865-70.

3. Varma AV, Malpani G, Agrawal P, et al. Clinicopathological spectrum of teratomas: An 8-year retrospective study from a tertiary care institute. Indian J Cancer 2017;54:576-9.

4. Gadducci A, Pistolesi S, Guerrieri ME, et al. Malignant Transformation in Mature Cystic Teratomas of the Ovary: Case Reports and Review of the Literature. Anticancer Res 2018;38:3669-75.

5. Kuo EJ, Sisk AE, Yang Z, et al. Adrenal Teratoma: a Case Series and Review of the Literature. Endocr Pathol 2017;28:152-8.

6. Wang F, Liu J, Zhang R, et al. CT and MRI of adrenal gland pathologies. Quant Imaging Med Surg 2018;8:853-75.

7. Ramakant $\mathrm{P}$, Rana C, Singh KR, et al. Primary adrenal teratoma: An unusual tumor - Challenges in diagnosis and surgical management. J Postgrad Med 2018;64:112-4.

8. Nadeem M, Ather MH, Sulaiman MN, et al. "Looks Can Be Deceiving": Adrenal Teratoma Causing Diagnostic Difficulty. Case Rep Urol 2015;2015:232591.

9. Ban A, Satapara J, Rathod K, et al. Teratoma involving adrenal gland - A case report and review of literature. Indian J Radiol Imaging 2019;29:472-6.

10. Narla SL, Jacob S, Kurian A, et al. Primary mature cystic teratoma with carcinoid mimicking an adrenal tumor: Report of a rare association and review of literature. Indian J Pathol Microbiol 2016;59:200-2.

11. Korobkin M, Giordano TJ, Brodeur FJ, et al. Adrenal adenomas: relationship between histologic lipid and CT and MR findings. Radiology 1996;200:743-7.

12. Hindman NM, Megibow AJ. One-Stop Shopping: DualEnergy CT for the Confident Diagnosis of Adrenal Adenomas. Radiology 2020;296:333-4.

Cite this article as: He C, Yang Y, Yang Y, Wang F, Hu J, Zhang J, Yin Y, Zeng L, Zhang T, Liu H. Teratoma of the adrenal gland: clinical experience and literature review. Gland Surg 2020;9(4):1056-1064. doi: 10.21037/gs-20-648
13. Namimoto T, Yamashita Y, Mitsuzaki K, et al. Adrenal masses: quantification of fat content with double-echo chemical shift in-phase and opposed-phase FLASH MR images for differentiation of adrenal adenomas. Radiology 2001;218:642-6.

14. Lam AK. Lipomatous tumours in adrenal gland: WHO updates and clinical implications. Endocr Relat Cancer 2017;24:R65-R79.

15. Angelousi A, Kyriakopoulos G, Nasiri-Ansari N, et al. The role of epithelial growth factors and insulin growth factors in the adrenal neoplasms. Ann Transl Med 2018;6:253.

16. Mhammedi WA, Ouslim H, Ouraghi A, et al. Adrenal myelolipoma: from tumorigenesis to management. Pan Afr Med J 2019;34:180.

17. Yang Y, Hu J, Zhang J, et al. Angiomyolipoma of the adrenal gland: clinical experience and literature review. Ann Transl Med 2020;8:654.

18. Maile CW, Rodan BA, Godwin JD, et al. Calcification in pulmonary metastases. Br J Radiol 1982;55:108-13.

19. Ghosh P, Santosa AC, Lin GY, et al. De novo calcification of liver and nodal metastases in prostate carcinoma. Prostate Cancer Prostatic Dis 2006;9:448-51.

20. Day K, Nikolaidis P, Casalino DD. Adrenal tuberculosis. J Urol 2012;187:285-6.

21. Del Borgo C, Urigo C, Marocco R, et al. Diagnostic and therapeutic approach in a rare case of primary bilateral adrenal tuberculosis. J Med Microbiol 2010;59:1527-9.

22. Guo YK, Yang ZG, Li Y, et al. Addison's disease due to adrenal tuberculosis: contrast-enhanced CT features and clinical duration correlation. Eur J Radiol 2007;62:126-31.

(English Language Editor: J. Chapnick) 